\title{
Electronically driven neutrons synthesis-generator with the magneto-optical flow seal
}

\author{
Mikhail Dolgopolov ${ }^{1, *}$, Albina Gurskaya ${ }^{1, * *}$, Andrey Privalov ${ }^{1, * * *}$, Vitaly Radenko ${ }^{2, * * * *}$, \\ Alexandr Radenko ${ }^{2, \dagger}$, and Vasily Svirkov ${ }^{2, \dagger}$ \\ ${ }^{1}$ Samara University, Academician Pavlov 1, 443011 Samara, Russia \\ ${ }^{2}$ Technological platform «Atomic and subatomic technologies» LLC. 605M, Academician Pavlov 1, \\ 443011 Samara, Russia
}

\begin{abstract}
We develop the description of a neutron generator construction for the synthesis of light nuclei. The design of the neutron generator with plasma target is given together with a description of the types of nuclear reactions that are implemented in it. The brief theoretical description of the ion multiphase flow in the synthesis generator is considered.
\end{abstract}

\section{Introduction}

Nowdays, neutron generators have applications in logging of oil and gas fields; logging of ore, including uranium, deposits; inspection systems remotely detecting of movement of explosives and nuclear materials; anti-cancer therapeutic systems and others [1]. Special attention of researchers and engineers is attracted by the possibility of synthesis of substances by neutron generators. Neutron generators with electronic control of ion flows in a magnetic field are used for nuclear synthesis too. In this case, it is important to create movement rules of ion flows. The study of the generation processes of short neutron pulses and the development of appropriate technical solutions aimed at creating neutron generators with certain time characteristics is a complex task that requires an effective solution.

To get into the range of nuclear forces in cases where charged particles or ions are used as flying particles, their kinetic energy must be sufficient to overcome the Coulomb repulsion of the nucleus. In cases where the energy of a charged particle or ion is less than the height of the Coulomb barrier, the probability of a nuclear reaction will tend to zero [2-5]. Particle beams of the required energies are easily obtained on modern accelerators. If the energy of the particle is insufficient to overcome the Coulomb barrier, it will experience an elastic scattering in the Coulomb field of the nucleus. This rule does not apply to light nuclei where the Coulomb barrier is low. To generate a stable nuclear fusion reaction, a number of conditions must be executed: 1) the total energy of the incoming particle and the target must be higher than the Coulomb repulsion energy; 2) The density of the incoming flow and the density

\footnotetext{
*e-mail: mvdolg@yandex.ru

**e-mail: a-gurska@yandex.ru

***e-mail: andreyprivalov1995@mail.ru

****e-mail: tp-aist@mail.ru

†e-mail: avradenko@yandex.ru

†e-mail: svirkov@mail.ru
} 
of the target for the pulse mode of operation must be $\geq 10^{22} \mathrm{~cm}^{-3}$; 3) Retention time in the magneto-optical chamber $\tau \geq 1 \mathrm{~s}$; 4) Energy of ions in the incoming stream $\geq 200 \mathrm{keV}$.

In this paper, we offer a synthesis generator of light nuclei with a magneto-optical control system of ion flows, which satisfies the above conditions.

\section{The neutron generator design}

Our synthesis generator uses of controlled flows of lithium and deuterium plasma. The synthesis section is combined magneto-optical system of alternating controlled magneto-optical subsystems. Synthesis occurs in the bombardment of a stream of deuterons and tritium plasma target. An upper dense layer of lithium or boron plasma with an average energy of $250 \mathrm{keV}$ is used for shielding drift neutrons. You can see the design of this generator on the Figure 1.

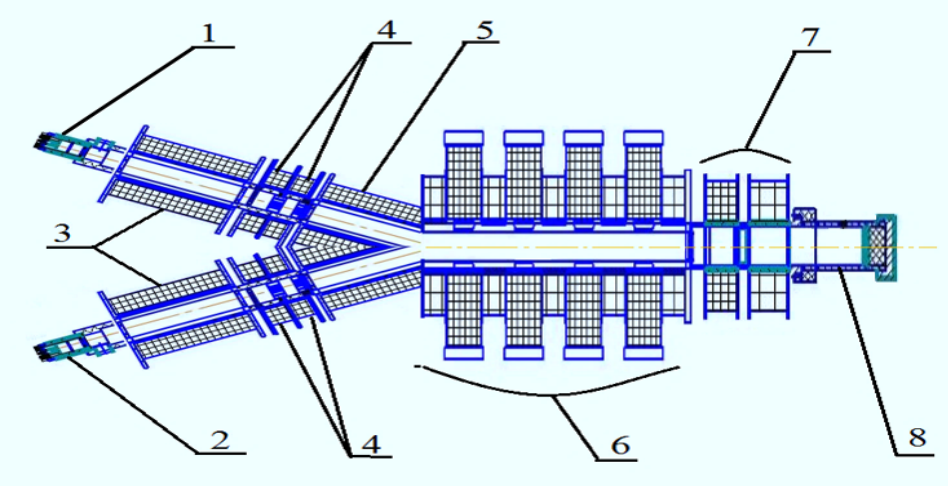

Figure 1. Neutron generator components: 1) Ionizer and deuterium and lithium; 2) Injector; 3) Linear pulsed accelerator; 4) Magneto-optical ion accumulator of ${ }^{2} \mathrm{H},{ }^{7} \mathrm{Li}$; 5) Accelerator-accumulator; 6) Magnetodynamic 4-cyclic camera; 7) Magneto-optical drive-limiter; 8) The system output neutrons.

The types of reactions for neutron generation are presented in the Table 1. These reactions are exothermic and can occur at arbitrarily low deuteron energies. In comparison with the generation of neutron pulses with a duration of $1-100 \mathrm{~ns}$ [6] using small-sized vacuum accelerator tubes, where it is possible at (D-T reaction, $300 \mathrm{kV}$ ) the neutron yield is $2 \cdot 10^{7}$ $\mathrm{n} / \mathrm{imp}$, the synthesis generator creates accelerated beams of lithium, deuterium and tritium ions, and then the formation of a dense ion or plasma target and a dense incoming flow allows the formation of neutron pulses from units of seconds to milliseconds from $2 \cdot 10^{10}$ to $2 \cdot 10^{14} \mathrm{n} / \mathrm{imp}$. The generation of the dense flow of protons, deuterium or tritium atoms for the neutrons synthesis on the ion-plasma target of deuterium, tritium or lithium occurs as the result of primary flow compaction and discretization by software-defined concentration and average energy of the flow. Flows are formed in strictly specified parameters: $T$ sequence period, $\mathrm{n}$ concentration and the frequency of discrete flows $\omega$.

\section{Modeling of ion and plasma multiphase flow}

Magnetodynamic $N$-cyclic camera synthesis consists of MOS magneto-optic systems - synthesis performed according to the scheme of combination of magnetic quadrupole lenses (MQL), providing a "strong" focus compression method discrete ion beams of hyperbolic 
Table 1. Types of reactions and energy parameters of synthesis

\begin{tabular}{|c|c|c|c|}
\hline Reaction & Energy, MeV & $\sigma_{\max }, \mathrm{b}($ region $\leq 1 \mathrm{MeV})$ & $E_{\text {imp.particle }}, \mathrm{MeV}$ \\
\hline \hline 1$) p+{ }^{6} \mathrm{Li} \rightarrow{ }^{4} \mathrm{He}+{ }^{3} \mathrm{He}$ & 4.0 & $10^{-4}$ & 0.3 \\
\hline 2$) p+{ }^{7} \mathrm{Li} \rightarrow{ }^{2} 4 \mathrm{He}+$ & 17.3 & $6 * 10^{-3}$ & 0.44 \\
\hline 3$) d+{ }^{6} \mathrm{Li} \rightarrow{ }^{7} \mathrm{Li}+p$ & 5.0 & 0.001 & 1.0 \\
\hline 4$) d+{ }^{6} \mathrm{Li} \rightarrow 2^{4} \mathrm{He}$ & 22.4 & 0.026 & 0.60 \\
\hline 5$) d+{ }^{7} \mathrm{Li} \rightarrow 2^{4} \mathrm{He}+n$ & 15.0 & $10^{-3}$ & 0.2 \\
\hline 6$) p+{ }^{9} \mathrm{Be} \rightarrow 2^{4} \mathrm{He}+d$ & 0.56 & 0.46 & 0.33 \\
\hline 7$) p+{ }^{9} \mathrm{Be} \rightarrow{ }^{6} \mathrm{Li}+{ }^{4} \mathrm{He}$ & 2.1 & 0.35 & 0.33 \\
\hline 8$) p+{ }^{1} 1 \mathrm{~B} \rightarrow 3^{4} \mathrm{He}$ & 8.7 & 0.6 & 0.675 \\
\hline 9$) p+{ }^{1} 5 \mathrm{~N} \rightarrow{ }^{1} \mathrm{CC}+{ }^{4} \mathrm{He}$ & 5.0 & $0.69($ at $1.2 \mathrm{MeV})$ & 1.2 \\
\hline
\end{tabular}

fields separated by a magneto-optical system shapers-limiters, performing the function of locking or crossing a discrete flow.

Effective length $L_{e f f}=\frac{1}{G_{0}} \int_{-\infty}^{+\infty} G(z) d z$. The field in the aperture of quadrupole lenses satisfies Maxwell's static equations: $\operatorname{rot} \vec{B}=0, \operatorname{div} \vec{B}=0$, static magnetic field potential $\vec{B}=\operatorname{grad} U_{0}$, satisfying the Laplace equation in cylindrical coordinates, the General solution of which is written as $U(r, \varphi)=\sum_{n=1}^{\infty} r\left(a_{n} \sin (n \varphi)+b_{n} \sin (n \varphi)\right)$. The initial parameters for the design calculation of a quadrupole lens determine the gradient of the focusing field, the distance from the lens axis to the pole and the number of ampere turns required to create a field with a given gradient, which is determined from the Maxwell integral equation: $\int_{\Gamma} \vec{H} d \vec{l}=\int_{S} \vec{j} d \vec{S}$. Calculating we obtain $\frac{G a^{2}}{\mu_{0}}=2 N I$, where $N I$ - number of ampere turns, $G$ -field gradient. The value of the transverse field gradient, which is related to the value of the current of the supply coils of the poles and $B_{p}$ magnetic induction at the pole $G=\frac{B_{p}}{r_{a}}$. Here $\mu_{0}$ is magnetic constant, $N I$ - number of ampere-turns in the exciting coil of the pole, $k-$ coefficient, what takes into account the physical properties of the material poles and yoke of the magnetic quadrupole lens; $r_{a}$ - the radius of the lens aperture.Offer to the attention of the development relies on the method of obtaining the controlled streams of ions or plasma, the realized magneto-optical shaper-drive, forming and modulatory accelerators for the formation of linear streams of plasma concentration required and the period of engagement. The technology of electronically controlled plasma synthesis of generators is a new method of obtaining the controlled streams of ions to obtain linear flow of ions or electrons selectable flow. That is, setting the laws of change of parameters (energy $E$, particle current $I$, concentration $n$, period $T_{s l}$ allows to form primary electronically controlled flows of charged particles-ions and electrons. The most common functional discretization in which the ion current, $j_{i}, n_{i}$ concentration, energy flow $E_{i}$ interconnected and change according to a certain law specified as a single function of sampling rate, $F\left(I_{i}, n_{i}, E_{i}\right)$.

The obtained discrete flows of ions and electrons formed by a group of particles with the same velocities and coordinates and concentrated in a small region of phase space forming clots or discrete. In the synthesis generator motion occurs in a magnetic solenoidal and quadrupole magnetic lenses MQL. In the electromagnetic field, the motion of the discrete is given by the Newton-Lorentz equation $\left(v=\frac{d R}{d t}\right)$ :

$$
\frac{d m v}{d t}=q(E+[v \times B]),
$$


where $R$ - radius-vector of the observed particle in the laboratory coordinate system; $m, q-$ particle mass and charge; $v$ - velocity; $E$ - vector of electric field intensity; $B$ - magnetic induction vector. Then for a two-component flow 1 can write so $\left(v_{i j}=\frac{d R}{d t}\right)$ :

$$
\frac{d m v_{i j}}{d t}=q\left(E+\left[v_{i j} \times B\right]\right)
$$

The movement of the discretes has the complex spatial nature, but if the speed lies in the symmetry plane, the motion is discrete to be flat. Quadrupole lenses are referred to as transverse lenses whose magnetic fields have a stronger effect on the movement of particles compared to longitudinal. For a discrete moving in the quadrupole lens in the plane $y=0$ the force $F_{x}=e v_{z} B_{y}$ acts, where $e$ - particle charge, $v_{z}$ - the longitudinal velocity of the particle is equal to the value of the total velocity at $y=0$. For a more rational solution we need to find the matrix function we need to find the matrix function $K\left(\frac{z}{z_{0}}\right)$. Transformation of the coordinates of the trajectory phase moments of each discret on the plane perpendicular to the axis with the coordinate $z_{0}$ at the entrance to the MQL in the plane $z_{1} \geq z$, where $z_{1}$ is the plane at the output. Then can be write:

$$
\frac{d K\left(\frac{z}{z_{0}}\right)}{d z}=P(z) K\left(\frac{z}{z_{0}}\right), \quad K\left(\frac{z}{z_{0}}\right)=\mathbf{E},
$$

where $\mathbf{E}$ - identity matrix. For each MQL, and their synthesis generator from 4 to 16 , in our case 8 , knowing the matrix transformation function can describe the dynamics of motion of the discrete throughout the synthesis chamber by a series of matrix transformations of the coordinates of its trajectory phase moments. By setting each thread a time interval $\tau_{i j}$ termines the parameter sequence $\left(k_{i}, k_{j}\right.$ of each discrete D. For each $\tau_{i j}$ rresponds to a set of values $U_{i j}, I_{i j}$, discrete flow $z i j$. $U_{i j}$ - matrix of values of the accelerating voltage of the accelerator-driver and $I_{i j}$ - matrix of values of the electric current of the accelerator-generator. Generally $U_{i j}, I_{i j}$ sponds to $P_{i j}$ - matrix of values of electric power for a given accelerating voltage of the accelerator-driver.

Then for the formation of a multiphase flow will take place:

$$
F_{i}(D)=\sin \omega t+\phi_{i}, F_{i}(D)=\sin \omega t+\phi_{n}, F_{j}(D)=\cos \omega t+\phi_{i}, F_{j}(D)=\cos \omega t+\phi_{n}
$$

where $F_{i}(D)$ - function of $i$-component, $F_{j}(D)$ - function of $j$-component, $\omega$ - repetition frequency discretes, $\phi_{i}, \phi_{n}$ - phase discrete flow.

One of the embodiments of a method of forming a discrete trajectory streams with different phase values substantially rectilinearly relative to the axis of MQL is quadrature sweep along the axes $\mathrm{x}, \mathrm{y}$ in the output accelerator-modulator. The MOS scan DAC is supplied with a deflecting voltage functionally with the given values, which allows forming at the entrance distributed in the space of discrete multiphase flows.

\section{Conclusion}

We looked at the light isotope synthesis generator with plasma target. An important part of the device is the magneto-optical system, which has been discussed by us theoretically. The device of our generator allows us to increase the number of neutrons up to $10^{15}$ per pulse.

According to the data on nuclear reactions the minimum energy of the incident flow must be at least the threshold energy for the reaction $p+{ }^{7} \mathrm{Li} \rightarrow 2^{4} \mathrm{He}+, 0.44 \mathrm{MeV}$ at an energy output of $17.3 \mathrm{MeV}$. In our case, the energy of the target and the energy of the incoming flow are equal. By specifying the number of ions $N_{L i}, N_{H}$ and taking $N_{L i}=N_{H}$, we find the 
energy required for a given number of ions. At the given that in 8 grams of $\mathrm{LiH}$ it contains $6.022 * 10^{23}$ of nucleons.

Acknowledgements. Authors are grateful to organizers of The XXIV International Workshop High Energy Physics and Quantum Field Theory for the attention and opportunities of discussing results for futher experimental development.

\section{References}

[1] Watterson J.I.W., International Atomic Energy Agency 31, 5-14 (2000)

[2] Brown I.G., The Physics and Technology of Ion Sources (John Wiley Sons, New York, 1989) 464

[3] Mesyats G.A., Pulsed power and electronics (Nauka, Moscow, 2004) 704

[4] Forrester A.T., Large ion beams: Fundamentals of generation and propagation (WileyInterscience, New York, 1988) 338

[5] Tyrsa V.E., Burtseva L.P. Tech. Phys. 48, 807-812 (2003)

[6] Didenko A.N. et al., Tech. Phys. 59, 904-910 (2014) 